\title{
O TREINAMENTO COM RESTRIÇÃO DO FLUXO SANGUÍNEO NO GANHO DE FORÇA E HIPERTROFIA EM IDOSOS
}

\section{ARTIGO DE REVISÃO}

SANTANA, Gustavo de Sousa ${ }^{1}$, CANTÃO, Elbert Wander²

SANTANA, Gustavo de Sousa. CANTÃO, Elbert Wander. 0 treinamento com restrição do fluxo sanguíneo no ganho de força e hipertrofia em idosos. Revista Científica Multidisciplinar Núcleo do Conhecimento. Ano. 06, Ed. 12, Vol. 07, pp. 3449. Dezembro de 2021. ISSN: 2448-0959, Link de acesso: https:/www. nucleodoconhecimento.com.br/educacao-fisica/treinamento-comrestricao, $\quad$ DOI: $\quad 10.32749 /$ nucleodoconhecimento.com.br/educacaofisica/treinamento-com-restricao

\section{RESUMO}

De características irreversíveis e inevitáveis, o processo de envelhecimento é um processo natural a todos os seres vivos. Juntamente com o avanço da idade, podese observar a falta de independência do idoso em atividades simples do dia a dia, como por exemplo, a marcha, subir escadas, descer e subir calçadas. A todo momento novas propostas e novos métodos de treinamentos surgem para tentar atenuar as perdas comuns das capacidades físicas oriundas da senilidade. Uma nova opção de treinamento que vem apresentando bons resultados é o treinamento com restrição de fluxo sanguíneo (TRFS). Neste contexto, o presente artigo, visa responder: será que essa nova metodologia de treinamento promove resultados no desenvolvimento de força e hipertrofia em idosos? Sendo assim, o objetivo geral desse trabalho foi averiguar se há evidências científicas que demostrem se o RFTS em idosos é seguro e se traz benefícios para a população idosa. Para isso, foi feita uma busca em artigos relacionados ao tema nas bases PubMed, SciELO e Google

\footnotetext{
${ }^{1}$ Graduação em Educação Física.

${ }^{2}$ Mestrando em Ciências Aplicadas à Saúde (UFJ-GO), Pós-graduação em Nutrição Humana e Saúde (UFLA-MG), Graduação em Educação Física (FAGAMMON-MG).
}

RC: 103516

Disponível em: https://www.nucleodoconhecimento.com.br/educacaofisica/treinamento-com-restricao 
Acadêmico, além de livros de fisiologia humana e do exercício. A coleta de dados foi realizada dentre os meses de abril e agosto de 2020. No total, foram encontrados 368 trabalhos de 1995 a 2020 e, após análise, 24 trabalhos foram selecionados para subsidiar a redação desse artigo. Os resultados obtidos sugerem que o treinamento com restrição de fluxo sanguíneo é tão efetivo quanto o treinamento com altas cargas quando o objetivo é a hipertrofia, porém, para o ganho de força, o treinamento com altas cargas se mostrou mais eficaz, fazendo com que a combinação entre os dois métodos seja a melhor opção para esta população.

Palavras-chave: restrição de fluxo sanguíneo, hipertrofia, Kaatsu, idosos.

\section{INTRODUÇÃO}

Quando tratamos do termo envelhecimento, é bastante difícil encontrar um termo único e específico para definir as pessoas que se encontram na terceira idade, mas pode-se basear em dados estatísticos para definir essa população. Segundo a Organização das Nações Unidas (ONU, 1982), em países desenvolvidos, são consideradas idosas as pessoas com 65 anos ou mais, enquanto nos países em desenvolvimento, são idosos aqueles com 60 anos ou mais (MEIRELES, 2007).

Para Meireles et al. (2007) para definir o idoso pode ser utilizado a idade funcional que é determinada a partir do desempenho funcional do indivíduo, ou pode ser usada a idade cronológica, que é contabilizada a partir dos anos vividos desde o nascimento. O segundo método é o mais popular, por ser mais fácil de mensurar, mas isso não quer dizer que seja o mais eficaz. O mesmo autor afirma que ao comparar dois indivíduos com mesma idade cronológica é possível notar variações funcionais, mentais e/ou físicas. Envelhecer é um processo fisiológico que pode ser demonstrado de forma individual e variada.

O envelhecimento é um processo único, inevitável e que tem como características a redução gradativa da capacidade de vários sistemas orgânicos, limitando o bom funcionamento de suas funções (MAIOR, 2013; MONTEIRO, 1999). 
Maior (2013) afirma que o envelhecimento é um processo inevitável, todas as pessoas irão passar por ele, e que nele serão perdidos padrões de funções metabólicas em comparação a um indivíduo mais novo, e o processo em si, é algo único para cada indivíduo, não podendo assim, ser definido de uma única e exclusiva forma, pois cada pessoa irá envelhecer de sua maneira.

A busca por uma melhor qualidade de vida se faz necessária e o treinamento de força aparece como solução para combater a perda de massa muscular que ocorre nos idosos. Porém, devido a intensidade dos treinamentos, falta de ajuste de variáveis e a dificuldade na realização das atividades propostas, pode-se ter uma baixa adesão dos idosos ao treinamento.

O treinamento com restrição de fluxo sanguíneo (TRFS) é uma técnica que utiliza manguitos para gerar uma pressão externa, reduzindo assim o fluxo sanguíneo arterial e venoso em certas regiões do corpo durante o exercício físico (PATTERSON, 2019). Um manguito externo é utilizado para que a restrição do fluxo sanguíneo seja alcançada levando a uma restrição parcial ou total do retorno venoso de acordo com o protocolo a ser utilizado (SALLES, 2020). Tal procedimento leva a alterações metabólicas e fisiológicas que podem ser benéficas para a hipertrofia muscular.

Para buscar alternativas para a falta de adesão dos idosos ao treinamento de força convencional, o objetivo do presente estudo é o de analisar os efeitos do TRFS na força e hipertrofia muscular em idosos. Devido a algumas características particulares, o presente artigo visa responder: será que essa nova metodologia de treinamento promove resultados no desenvolvimento de força e hipertrofia em idosos?

Foi realizada uma pesquisa bibliografia baseada na fenomenologia. Segundo seus objetivos, caracteriza-se como descritiva e de acordo com Gil (2008) são as pesquisas que mais aprofundam o conhecimento com a realidade, por dizer o motivo e a razão das coisas.

RC: 103516

Disponível em: https://www.nucleodoconhecimento.com.br/educacaofisica/treinamento-com-restricao 
Para a coleta de dados foram utilizadas as bases cientificas eletrônicas: PubMed, SciELO, Google Acadêmico dentre outras, além da utilização de artigos e livros de Fleck e Kraemer, Alex Souto Maior, Belmiro Freitas De Salles, Roberto Simão, Baechle. Foram selecionados artigos nas bases de dados eletrônicos, utilizando como principal caráter de busca as seguintes palavras chaves: "Blood Flow Restricted", "Kaatsu", "Hypertrophy", "Seniors". A seleção foi realizada entre os meses de abril a agosto de 2020. Um total de 368 artigos foram pré-selecionados e como critérios de inclusão, foram utilizados artigos de 1995 até 2020 e que apresentaram conceitos gerais e específicos sobre os efeitos fisiológicos desse método de treinamento, além da literatura básica sobre processos fisiológicos. Como critérios de exclusão, foram retirados artigos que não contemplavam exatamente o TRFS e que não tinham uma relação direta entre as palavras-chave. No final, 24 trabalhos foram utilizados nessa revisão. A análise de dados segue um padrão quanti-qualitativo de forma que poderemos analisar quais os possíveis ganhos, tanto de hipertrofia quanto de força e potência nos indivíduos analisados.

\section{ENVELHECIMENTO E TREINAMENTO RESISTIDO}

A perda da força muscular está entre as alterações negativas mais importantes em idosos e associa-se diretamente à dependência funcional (MAIOR, 2013).

Segundo Evans (1995), a nomenclatura sarcopenia é derivada do grego "pobreza da carne", por estar relacionada a perda de força e massa muscular em decorrência da idade, com posterior comprometimento funcional, tendo impacto na realização das atividades da vida diária (DUTTA, 1997; MONTEIRO, 1999). A sarcopenia afeta a capacidade neuromuscular com o avanço da idade, acompanhado da perda involuntária de força e massa muscular (MAIOR, 2013; ROSENBERG, 1997).

Por deteriorar a homeostase biológica, a sarcopenia representa uma vulnerabilidade fisiológica relacionada à idade. Em idosos a força muscular é de vital importância para uma vida independente e funcional. Por isso, a perda de massa muscular e de força pode levar a um quadro dependência funcional. Vários possíveis mecanismos

RC: 103516

Disponível em: https://www.nucleodoconhecimento.com.br/educacaofisica/treinamento-com-restricao 
conduzem à sarcopenia, dentre eles, a redução de moto neurônios, de secreções hormonais, desnutrição e atrofia pelo estilo de vida sedentário (MAIOR, 2013).

Em uma pesquisa realizada por Maior (2013), visando observar a sarcopenia, foram estudados idosos com idade entre 64 e 93 anos, sendo 195 do sexo feminino e 142 do sexo masculino. A pesquisa mostrou que $22,6 \%$ das mulheres apresentam sarcopenia e os homens, 26,8\%, esses com idade de até 65 anos. Em um outro grupo, cuja idade foi a partir de 80 anos, mulheres apresentaram taxa de $31 \%$ e homens $52,9 \%$.

Segundo Maior (2013), a redução de massa muscular associa-se a outras perdas como a de nitrogênio, cálcio, água e minerais ósseos.

Com o envelhecimento há um decréscimo dos minerais ósseos, aumentando a porosidade dos ossos, tornando-os mais frágeis. Esse processo de deterioração pode ser tão grande, que uma simples atividade da vida cotidiana pode causar uma fratura óssea, particularmente no quadril, coluna ou punho (SIMÃO, 2004).

Ainda de acordo com Maior (2013) se associarmos o envelhecimento com a inatividade muscular é possível constatar que grande parte das atrofias musculares são encontradas nas fibras tipo II, essas que são exigidas para o treinamento de força. A sarcopenia está presente em indivíduos sedentários, porém, pode também ser vista em indivíduos ativos ao longo da vida, sendo assim, urge a necessidade de uma atenção à saúde pública.

Silva (2006) relatam que atualmente o meio mais utilizado para o diagnóstico de sarcopenia é a densitometria óssea de corpo total para a avaliação da composição corporal (massa óssea, massa magra e massa adiposa total).

A densitometria óssea de corpo inteiro também conhecida mais popularmente como DEXA, é um exame de padrão ouro para analisar a composição corporal, não invasivo, muito simples para o paciente e tem duração média de 10 a 15 minutos. 
Ainda não existe um consenso único sobre como definir a sarcopenia, doença ou processo natural de envelhecimento, pois a perda de massa muscular pode vir a acontecer mesmo durante o envelhecimento saudável, assim sendo, a sarcopenia deveria ser considerada doença somente quando levar a inaptidão funcional (MAIOR, 2013).

Alguns autores só colocam a sarcopenia como doença se ela estiver associada a alguma limitação funcional, pois a sarcopenia faz parte do processo de envelhecimento natural e fisiológico (SILVA, 2006).

Desta maneira, a sarcopenia pode ser tratada como um meio de definir a debilitação funcional de um indivíduo mais velho, em outras palavras, não é tratada como uma enfermidade, mas sim como um processo de limitação da mobilidade do idoso.

Para Fry et al. (2010) a perda de massa muscular esquelética durante o envelhecimento aumenta o risco de quedas e dependência. $O$ exercício resistido (ER) é uma técnica de reabilitação eficaz que pode melhorar a massa e a força muscular; no entanto, indivíduos mais velhos são resistentes à estimulação da síntese de proteínas musculares com ER de alta intensidade tradicional.

De acordo com Maior (2013) os exercícios resistidos aparecem como fatores consideráveis para minimizar e retardar o processo de sarcopenia com significantes respostas neuromusculares por meio do aumento da capacidade contrátil dos músculos esqueléticos.

A força muscular, uma habilidade complexa, é caracterizada como a tensão gerada por um músculo ou um grupo muscular contra uma resistência externa. O treinamento de força (TF) é utilizado como um meio efetivo de incremento da força muscular e melhoria do estado funcional em todas as populações. Por isso, faz-se necessário o uso de sobrecargas na prescrição do treinamento, melhorando o desempenho físico através do aumento da força e potência muscular (MAIOR, 2013).

RC: 103516

Disponível em: https://www.nucleodoconhecimento.com.br/educacaofisica/treinamento-com-restricao 
Os termos treinamento resistido e treinamento de força possuem grande abrangência de modalidades de treinamento: exercícios corporais com pesos, uso de tiras elásticas, pliométricos e corrida em ladeiras são alguns exemplos. Já o termo treinamento com pesos, também conhecido como musculação, costuma-se referir apenas ao treinamento resistido com pesos livres ou com algum tipo de equipamento de treinamento com pesos (FLECK; KRAEMER, 2017).

A literatura crescentemente vem demonstrando a efetividade do treinamento resistido na melhora da massa muscular, força, resistência e equilíbrio em indivíduos idosos. Segundo Giallauria (2015) o treinamento de resistência pode aumentar enormemente a taxa de síntese de proteína muscular mista em idosos fisicamente frágeis. Após 3 meses de exercícios de levantamento de peso, as taxas de síntese de proteína contrátil muscular nesses idosos sedentários aumentaram bastante.

Homens mais velhos treinados têm a força de extensão do joelho duplicada em comparação a indivíduos da mesma faixa etária sedentários, isso também tem sido observado similarmente em mulheres de idade avançada. Em um estudo que incluiu homens e mulheres com idade entre 87 e 96 anos foi demonstrado que após apenas 8 semanas de treinamento de força, esse grupo obteve melhoras na velocidade do andar, potência para subir escadas, equilíbrio e atividade espontânea (SIMÃO, 2004)

O treinamento de força vem se mostrando como um tipo seguro de exercício mesmo para os idosos frágeis. Idosos de ambos os sexos têm se beneficiado com essa prática. Mesmo após vários meses de treino, os voluntários continuaram a mostrar melhoras. Como adaptações funcionais ao treinamento de força temos endurance aumentada para caminhada, tempo de equilíbrio maior, menor tempo para subir escadas e redução no risco de quedas. Todos esses benefícios fazem com que o idoso tenha uma vida mais autônoma, funcional e independente (FRONTERA, 1997).

RC: 103516

Disponível em: https://www.nucleodoconhecimento.com.br/educacao-

fisica/treinamento-com-restricao 
Evidências de estudos apontam que o treinamento de força regular também mostra efeitos anabólicos em idosos. A tomografia computadorizada e a análise da biópsia muscular já mostraram evidências positivas na hipertrofia muscular de homens mais velhos participantes de um programa de treinamento de força de alta intensidade. Outros estudos relataram que o treinamento de força pode incrementar a retenção de nitrogênio (demonstra um efeito positivo no metabolismo da proteína muscular) e melhor equilíbrio energético, oriundo de um aumento na taxa de metabolismo basal em idosos de ambos os sexos praticantes de treinamento de força (SIMÃO, 2004).

Segundo Simão (2004) mesmo que o efeito relacionado a parte óssea dependa de vários outros fatores, há registros de que o treinamento de força proporcione efeito positivo na saúde óssea de indivíduos mais velhos. Esse tipo de treinamento, se realizado de forma regular, pode vir a compensar as perdas ósseas relacionadas a idade, levando a manutenção ou ao aumento da densidade mineral óssea.

Grande parte da população idosa é relutante em participar do treinamento de força, pois, sua maioria acredita que, pela idade avançada não será capaz de realizar ou até mesmo de obter mudanças corporais significantes, tanto no aspecto de composição corporal como no aspecto fisiológico. Baechle (2013) realizou um estudo de 14 semanas, em pacientes frágeis de lares de idosos, com média de idade de 90 anos, o que mostrou modificações positivas na composição corporal. $O$ programa de treinamento de força de apenas 10 minutos (uma série em cinco exercícios), duas vezes por semana, produziu ganhos médios de 1,7 kg de massa magra (muscular) e perda de $1,3 \mathrm{~kg}$ de gordura.

\section{TREINAMENTO COM RESTRIÇÃO DE FLUXO SANGUÍNEO (TRFS)}

O TRFS, ou treinamento Kaatsu (que significa, treinamento com pressão adicional) é uma técnica que utiliza manguitos para gerar uma pressão externa, reduzindo assim o fluxo sanguíneo arterial e venoso em certas regiões do corpo durante o exercício físico (PATTERSON, 2019). A oclusão vascular é definida como a oclusão focal de 
veias e artérias que promove a diminuição do fluxo sanguíneo muscular (KAWADA, 2005).

A restrição do fluxo sanguíneo é alcançada utilizando pressão externa através de um manguito com pressão suficiente para que ocorra restrição parcial ou total do retorno venoso de acordo com o protocolo a ser utilizado (SALLES, 2020).

A pressão para oclusão arterial (AOP) deverá ser definida com intuito de atender as necessidades específicas para cada pessoa que realizará o treinamento, assim como a largura e tamanho do manguito, pois diferentes tamanhos e comprimentos da musculatura alteram a pressão necessária para realizar a oclusão. No estudo de Patterson (2019) é sugerido que se utilize a oclusão de maneira personalizada, baseada em \% da AOP total e mostra-se mais eficiente se utilizado de $40 \%$ a $80 \%$ da AOP total. A largura do manguito também deve ser levada em consideração pois um manguito mais estreito exigirá uma pressão mais alta. É sabido também que uma pressão de $40 \%$ da AOP não necessariamente corresponde a uma redução efetiva de $40 \%$ do fluxo sanguíneo. Neste mesmo estudo verificou-se que a aplicação em \% da AOP, em três manguitos de tamanhos diferentes, produziu uma redução semelhante no fluxo sanguíneo em repouso, o que mostrou que um manguito mais largo exigia uma menor pressão se comparado a um manguito estreito, esse deveria ser inflado a uma pressão superior para que fosse possível atingir a mesma \% da AOP, para que obtivesse uma restrição semelhante com ambos os manguitos.

Quanto maior o manguito, menos pressão será exigida para uma restrição efetiva do fluxo sanguíneo, levando em consideração que manguitos grandes demais podem vir a atrapalhar a realização dos movimentos, dificultando ou atrapalhando a execução dos exercícios.

Deste modo parece existir uma relação entre a pressão aplicada durante o exercício e a carga relativa levantada durante o exercício resistido. Para cargas correspondentes a $20-40 \%$ do nível máximo de força de um indivíduo (baseado no teste de uma repetição máxima) provavelmente maximizará o crescimento e a força 
muscular (LIXANDRAO et al., 2015 ; COUNTS et al., 2016 ). Cargas de aproximadamente $20 \%$ de uma repetição máxima requerem uma pressão mais alta (aproximadamente $80 \%$ de AOP) para estimular o crescimento muscular (LIXANDRAO et al., 2015), no entanto, outros estudos são necessários para confirmar isso (PATTERSON, 2019).

Um estudo conduzido por Centner (2018) buscou avaliar os resultados do treinamento com restrição de fluxo sanguíneo com baixas cargas e o treinamento de caminhada com restrição de fluxo sanguíneo e verificou-se que a restrição do fluxo sanguíneo gerou melhorias significativas no ganho de força muscular. O mesmo aconteceu com a massa muscular: ganhos maiores comparando com e sem a restrição do fluxo sanguíneo. Quando analisado os ganhos hipertróficos, o treinamento com restrição de fluxo sanguíneo com baixas cargas gerou ganhos semelhantes ao do treinamento convencional com altas cargas. Porém, os ganhos de força foram menores.

Devido à insuficiência de dados, não é possível concluir sobre os efeitos do treinamento de baixa carga com restrição de fluxo sanguíneo na massa muscular em comparação com o treinamento de baixa carga isolado. Curiosamente, mesmo durante intensidades tão baixas quanto a caminhada, o treinamento com restrição de fluxo sanguíneo melhora as adaptações de força e massa muscular em indivíduos mais velhos em comparação com a caminhada normal (CENTNER, 2018).

Cook (2019) sugeriu que o treinamento com restrição de fluxo sanguíneo pode vir a divergir nas adaptações encontradas entre ganho de massa muscular e força. $\mathrm{O}$ autor realizou um estudo com um grupo de vinte e um idosos que possuíam idades entre 67 e 90 anos. O grupo de alta carga apresentou uma maior progressão de carga no treinamento de 12 semanas principalmente na extensão de joelho.

O TRFS vem demonstrando adaptações positivas com cargas bem baixas $(<50 \% 1$ RM) para ganhos de força e hipertrofia. Esse percentual fica bem abaixo dos 
percentuais que geralmente são utilizados no treinamento convencional da musculação (>60\% de $1 \mathrm{RM}$ ) para os mesmos objetivos (SALLES, 2020).

Segundo Centner (2018) o estresse metabólico é o mecanismo mais plausível que tem sido relatado durante os estudos utilizando a restrição de fluxo sanguíneo. Vários estudos mostraram que a hipóxia intramuscular, aliada ao estresse metabólico, foram influenciadores para a fadiga das fibras musculares levando assim ao recrutamento das demais fibras durante o treinamento.

O estresse metabólico, com o aumento do $\mathrm{Ph}$ intramuscular em decorrência da hidrólise de ATP, aumento da hiperemia muscular e consequente ruptura sarcolema, estão entre outros respostas do TRFS (NASSER; NETO, 2017).

De acordo com Biazon (2019) pode-se aceitar que a hipertrofia se dá por dois mecanismos, a tensão mecânica e o estresse metabólico, cujos programas de treinamento resistido com alta carga em torno de $80 \%$ de $1 \mathrm{RM}$ podem ativar os dois mecanismos citados. Por outro lado, o treinamento resistido com restrição de fluxo sanguíneo tem mostrado respostas semelhantes no aumento da área de secção transversa muscular, tendo como principal mecanismo o estresse metabólico.

Foi demonstrado que o TRFS produz maiores alterações na concentração de desoxihemoglobina no sangue, isto é, marcador substituto do estresse metabólico (BIAZON, 2019). Esse treinamento gera hipóxia tecidual, aumento do acúmulo de metabólitos (estresse metabólico), aumento do recrutamento de fibras de contração rápida (Tipo II), da produção hormonal sistêmica e localizada, do inchaço celular e da produção de óxido nítrico. Esses e outros efeitos podem favorecer a hipertrofia (SALLES, 2020).

Alguns estudos apontam que uma das respostas ligadas a hipertrofia, após 2 semanas de treinamento, pode estar relacionada diretamente ao alto estresse induzido pelo treinamento com restrição de fluxo sanguíneo (BFR), o que indica que o estresse mecânico passa a ser relativo quando se pensa em hipertrofia. Outros estudos, porém, tem demonstrado que a hipertrofia, no período citado, é

RC: 103516

Disponível em: https://www.nucleodoconhecimento.com.br/educacaofisica/treinamento-com-restricao 
consequência do edema muscular gerado pelo treinamento resistido fazendo com que, assim, sejam necessários exames específicos para determinar a resposta real a hipertrofia (BIAZON, 2019).

O método de treinamento com restrição de fluxo sanguíneo pode ser bem suportado por todos que se disporem a executá-lo. Fry (2010) utilizou um questionário para avaliar a relação de esforço percebido durante a aplicação do método e obtiveram uma média de 7 a 8 em uma escala de 0 a 10.

FRY et al. (2010) demonstram que a utilização da restrição de fluxo sanguíneo melhora a sinalização do mTOR (alvo mamífero da rapamicina) da primeira até a terceira hora após a realização do treinamento, assim como mostrado na FIGURA 1 abaixo.

Figura 1 - Sinalização da mTOR em 3 horas pós-treinamento com restrição de fluxo sanguíneo.

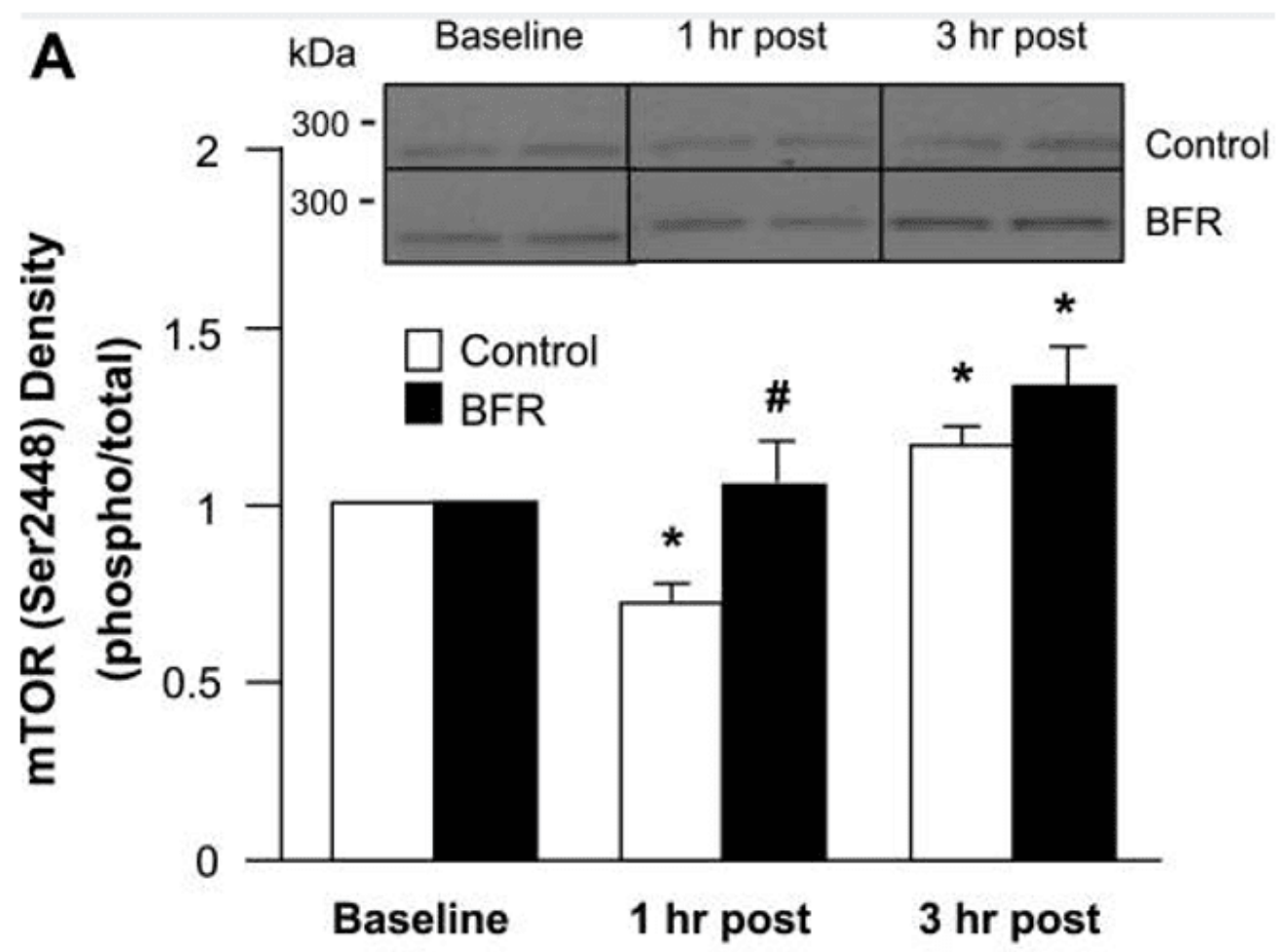

Fonte: FRY et al. (2010).

RC: 103516

Disponível em: https://www.nucleodoconhecimento.com.br/educacaofisica/treinamento-com-restricao 
Como observado na FIGURA 1, podemos verificar em preto os marcadores de mTOR utilizando o método de restrição do fluxo sanguíneo. Em branco, o grupo controle ou método de treinamento tradicional e suas diferenças pós treino dos níveis de mTOR.

\begin{abstract}
Estudos mostram que a utilização da oclusão vascular combinada ao TF de baixa intensidade (entre $20 \%$ e $40 \%$ de 1 RM), também chamado de Kaatsu training, contribui para o estado de hipóxia da musculatura e o acúmulo metabólico, que colaboram para o aumento de fatores anabólicos, como: aumento da síntese do hormônio do crescimento; aumento da atividade do alvo da rapamicina em mamíferos (mTOR), responsável pela realização da síntese de proteínas; aumento da atividade da enzima óxido nítrico sintase 1 (NOS-1); recrutamento precoce das fibras musculares do tipo II e aumento da expressão da proteína de choque térmico 72 (HSP72), fatores primordiais para ganhos significativos de hipertrofia muscular e força muscular em atletas, indivíduos fisicamente ativos e indivíduos enfermos (MAIOR, 2013, apud KAWADA, 2005; FUJITA, 2007; LOENNEKE, 2010; SATOH, 2011).
\end{abstract}

Treinos com 30\% de uma repetição máxima ( $1 \mathrm{RM}$ ) levados até a falha concêntrica promoveram um aumento na atividade da via Akt/mTOR e na sinalização de algumas proteínas, como a P70S6K e Erk 1/2 (NASSER; NETO, 2017; BURD, 2010). Maiores expressões dessas proteínas estão associadas a hipertrofia muscular (TERZIS, et al., 2008).

\title{
CONSIDERAÇÕES FINAIS
}

A questão inicial era encontrar evidências científicas se o TRFS traria os mesmos resultados positivos para os idosos como em outras faixas etárias. Sendo assim, retomando a questão norteadora: será que essa nova metodologia de treinamento promove resultados no desenvolvimento de força e hipertrofia em idosos? Concluímos que, após analisar as respostas da mTOR, MAPK (proteínas quinases ativadas por mitógenos) e MPS (síntese de proteínas musculares) ao treinamento com restrição de fluxo sanguíneo, pode-se dizer que este método de treinamento promove ganhos hipertróficos semelhantes, se não melhores, quando comparado ao treinamento tradicional com altas cargas podendo ser induzidos em questão do

RC: 103516

Disponível em: https://www.nucleodoconhecimento.com.br/educacaofisica/treinamento-com-restricao 
estresse metabólico obtido em função da isquemia gerada por estímulos mecânicos externos, tendo como benefício, ao mesmo tempo, uma menor percepção de esforço realizado e gerando uma menor dor pós treino, tornando o treinamento mais "confortável", com menores chances de ocorrer tensão mecânica, menor risco de over training ou lesões osteomusculares, sendo benéfico a população idosa.

Em relação ao ganho de força, foi possível observar que se comparado ao treinamento com altas cargas, os ganhos de força foram menores, o que se pode concluir que, para o ganho de força, e consequentemente potência, o treinamento com cerca de $80 \%$ de 1 RM passa a ser mais efetivo. Consequentemente, se considerado a capacidade de locomoção, o treinamento tradicional com alta carga seria mais indicado para a população idosa pelo fato de trabalhar com o percentual de sua repetição máxima e não com uma carga pré-estabelecida.

Deste modo, baseado nos diversos estudos, sugere-se que a melhor forma para o ganho de força e hipertrofia em idosos, possa ser trabalhada de maneira combinada e periodizada com os dois métodos de treinamentos citados (treinamento com alta carga e treinamento com restrição de fluxo sanguíneo), visando assim, uma melhoria em aspecto global do quadro de evolução de força e hipertrofia em idosos.

Recomenda-se que estudos sobre o tema proposto nesta pesquisa seja fundamento de treinamento para o corpo profissional de educação física, em razão que poucos profissionais têm conhecimento para a aplicação do treinamento de força com restrição do fluxo sanguíneo. Sugere-se que pesquisas futuras sejam realizadas com intuito de obterem mais dados pertinentes ao tema abordado, com fins de melhoria na qualidade de vida dos idosos.

\section{REFERÊNCIAS}

BAECHLE, T. R.; WESTCOTT, W. L.; Treinamento de força para a terceira idade. São Paulo: ARTMED Editora, 2013. Tradução: Cássio Victoria Ruas; Eurico Nestor Wilhelm Neto; Regis Radaelli. 
BIAZON, T. et al. The Association Between Muscle Deoxygenation and Muscle Hypertrophy to Blood Flow Restricted Training Performed at High and Low Loads. Frontiers in Physiology. 2019. Disponível em: <https://www.ncbi.nlm.nih.gov/pmc/articles/PMC6479177/> Acesso em: 12 maio 2020.

BURD, N.C.; WEST, D.W.D.; STAPLES, A.W.; ATHERTON, P.J.; BAKER, J.M.; MOORE, D.R. et al. Low-load high volume resistance exercises stimulates muscle protein synthesis more than high-load low volume resistance exercise in young men. PloS One. Num. 5. Vol. 8. 2010. p.e12033.

CENTNER, C. et al. Effects of Blood Flow Restriction Training on Muscular Strength and Hypertrophy in Older Individuals: A Systematic Review and Meta-Analysis. Sports Medicine (Auckland, N.z.). 2019. Disponível em: <https://www.ncbi.nlm.nih.gov/pmc/articles/PMC6349784/> Acesso em: 12 maio 2020.

COOK, V. B.; CLEARY, C. J. Progression of Blood Flow Restricted Resistance Training in Older Adults at Risk of Mobility Limitations. Frontiers in Physiology. 2019. Disponível em: <https://www.ncbi.nlm.nih.gov/pmc/articles/PMC6582311> Acesso em: 23 maio 2020.

COUNTS B. R., DANKEL S. J., BARNETT B. E., KIM D., MOUSER J. G., ALLEN K. $M$., et al. (2016). Influence of relative blood flow restriction pressure on muscle activation and muscle adaptation. Muscle Nerve 53 438-445. 10.1002/mus.24756.

DUTTA, C. Significance of sarcopenia in the elderly. J. Nutr., v. 127, n. 5, 1997.

EVANS, W. J. What is sarcopenia? J. Gerontol. A. Biol. Sci. Med. Sci., v. 50, n. 5-8, 1995.

RC: 103516

Disponível em: https://www.nucleodoconhecimento.com.br/educacao-

fisica/treinamento-com-restricao 
FLECK, S. J.; KRAEMER, W. J.; Fundamentos do treinamento de força muscular. São Paulo: ARTMED Editora, 2017. Tradução: Jerri Luiz Ribeiro; Regina Machado Garcez.

FRONTERA, Walter R. A importância do treinamento de força na terceira idade. Rev. Bras. Med. Esporte, Niterói, v.3, n.3, p. 75-78, set. 1997. Disponível em<http://www.scielo.br/scielo.php?script=sci_arttext\&pid=S1517-

$86921997000300003 \&$ lng=pt\&nrm=iso $>$. Acesso em: 09 maio 2020

FRY, C. S. et al. Blood flow restriction exercise stimulates mTORC1 signaling and muscle protein synthesis in older men. Journal of applied physiology. 2010. Disponível em:< https://www.ncbi.nlm.nih.gov/pmc/articles/PMC2867530/>Acesso em: 12 maio 2020.

GIALLAURIA, F., CITTADINI, A., SMART, N. A., \& VIGORITO, C. (2016). Resistance training and sarcopenia. Monaldi Archives for Chest Disease.84(1-2). https://doi.org/10.4081/monaldi.2015.738

GIL, Antônio Carlos. Métodos e Técnicas de Pesquisa Social. 6. ed. - São Paulo: Atlas, 2008. Disponível em: https://ayanrafael.files.wordpress.com/2011/08/gil-a-cmc3a9todos-e-tc3a9cnicas-de-pesquisa-social.pdf Acesso em: 26 abril 2020

KAWADA, S.; ISHII, N. Skeletal muscle hypertrophy after chronic restriction of venous blood flow in rats. Med. Sci. Sports Exerc., v. 37, p. 1144-50, 2005.

LIXANDRÃO M. E., UGRINOWITSCH C., BERTON R., VECHIN F. C., CONCEIÇÃO M. S., DAMAS F., et al. (2018). Magnitude of muscle strength and mass adaptations between high-load resistance training versus low-load resistance training associated with blood flow restriction: a systematic review and meta-analysis. Sports Med. 48 361-378. 10.1007/s40279-017-0795-y

MAIOR, Alex Souto. Fisiologia dos exercícios resistidos [recurso eletrônico] / Alex Souto Maior. - São Paulo: Phorte, 2013. recurso digital: il. 
MEIRELES, Viviani Camboin et al. Características dos idosos em área de abrangência do Programa Saúde da Família na região noroeste do Paraná: contribuições para a gestão do cuidado em enfermagem. Saúde e sociedade. São Paulo, v. 16, n. 1, p. 69-80, Apr. $2007 . \quad$ Available from $<$ http://www.scielo.br/scielo.php?script=sci_arttext\&pid=S010412902007000100007\&Ing=en\&nrm=iso > . access on 14 Sept. 2020. https://doi.org/10.1590/S0104-12902007000100007.

MONTEIRO, W. D. et al. Força muscular e características morfológicas de mulheres idosas praticantes de um programa de atividades físicas. Rev. Bras. Ativ. Fís. Saúde, v. 4, n. 1, 1999.

NASSER, Igor; NETO, Victor G. C. Treinamento de força com baixas cargas e alto volume para hipertrofia: análise de parâmetros moleculares. Revista Brasileira de Prescrição e Fisiologia do Exercício. São Paulo. v.11. n.68. p.610-619. Set./out. 2017. Disponível em <http://www.rbpfex.com.br/index.php/rbpfex/article/view/1162> Acesso em: 06 Outubro 2020

ROSENBERG, I. H. Sarcopenia: origins and clinical relevance. J. Nutr., v. 127, n. 5, 1997.

PATTERSON, S. D. et al. Blood Flow Restriction Exercise: Considerations of Methodology, Application, and Safety. Frontiers in Physiology 2019. Disponível em: <https://www.ncbi.nlm.nih.gov/pmc/articles/PMC6530612/> Acesso em: 26 abril 2020.

SALLES, Belmiro Freitas de. Métodos de treinamento para força e hipertrofia: da teoria à prática. Belo Horizonte: Livro na Mão, 2020.

SILVA, Tatiana Alves de Araújo et al. Sarcopenia associada ao envelhecimento: aspectos etiológicos e opções terapêuticas. Rev. Bras. Reumatol., São Paulo, v. 46, n. 6, p. 391-397, Dec. $2006 . \quad$ Available from <http://www.scielo.br/scielo.php?script=sci_arttext\&pid=S0482-

RC: 103516

Disponível em: https://www.nucleodoconhecimento.com.br/educacaofisica/treinamento-com-restricao 
$50042006000600006 \&$ lng $=$ en $\&$ nrm $=$ iso $>$.

access

on 17

Sept.

2020. https://doi.org/10.1590/S0482-50042006000600006.

SIMÃO, R. Fisiologia e prescrição de exercícios para grupos especiais. São Paulo: Phorte Editora, 2004.

Enviado: Julho, 2021.

Aprovado: Dezembro, 2021. 